\title{
Alternative mathematical method for calculating biological age
}

\author{
Anatoly V. Pisaruk \\ D.F. Chebotarev Institute of Gerontology NAMS of Ukraine, Kiev, Ukraine \\ https://doi.org/10.47855/jal9020-2021-2-2
}

Correspondence: avpisaruk54@gmail.com

Received: 15.02.2021; Accepted: 25.03.2021; Published: 06,06.2021

\begin{abstract}
To eliminate the shortcomings of calculating biological age by the multiple regression method, an alternative mathematical approach is proposed. This method consists in calculating the average value of the ratio of the values of the indicators of the examined person to the average population values of these indicators. Comparison of this method with the regression method on the data set of healthy people showed that it eliminates the disadvantages of the regression method, but gives a slightly lower accuracy in assessing biological age.
\end{abstract}

Keywords: biological age; mathematical method

The main mathematical method for calculating biological age (BA) is the method of stepwise multiple regression [1-6]. But it has a number of limitations and disadvantages.

1. To receive a regression equation linking the age of people with biomarkers of aging, complete data are required for all indicators for each person.

2. The number of people surveyed should be $2-3$ orders of magnitude more than the number of indicators selected for calculating the regression equation.

3. To calculate BA for an individual according to the received regression equation, it is necessary to know the values of all indicators included in the formula.

4. The regression model, by its nature, overestimates BA in young people and underestimates in old people.

It is difficult or even impossible to recievei complete data (to carry out all tests) for each person in practice. Therefore, alternative methods for calculating BA have been proposed. One of these approaches is a method based on calculating the average value of the ratio of the values of the indicators of the examined person to the average population values of these indicators [7]. This method does not have all the listed disadvantages of the regression method. The BA formula for this method is recieved as follows:

1. A battery of tests is created to assess BA by selecting indicators that correlate well with age and weakly with each other.

2. Find the average values of each indicator in different age groups from 20 to 80 years old, usually by decades.

3. The rate of aging (AR) of a person is calculated as the sum of the ratios of the values of each indicator to the average values of the corresponding indicators in the age group to which the given person belongs, divided by the number of indicators.

$$
\mathrm{AR}=\Sigma(\mathrm{I}(\mathrm{i}) / \operatorname{Im}(\mathrm{i})) / \mathrm{N} ; \mathrm{BA}=\mathrm{CA} \times \mathrm{AR}
$$

CA - chronological age; 
I (i) - value of the I ${ }^{\text {th }}$ indicator;

$\operatorname{Im}(\mathrm{i})$ - average value of the $\mathrm{i}^{\text {th }}$ indicator in a group of people of the same age;

$\mathrm{N}$ - the number of indicators used in the calculation.

This approach makes it possible to use incomplete databases or data from other researchers who studied age-related changes in individual indicators to obtain average values in different age groups, which is an advantage of this approach. It is possible to calculate BA and the aging rate of a particular person even if not all tests have been performed. This, of course, reduces the validity of such an estimate.

To receive a more accurate assessment of BA by this method, it is possible to calculate the ratio of the indicator value not with its average value in the corresponding age group, but with the calculated population average value of the indicator at a given age $(\mathrm{Im})$. Im is calculated by the BM regression equation with age in healthy people.

$$
\operatorname{Im}=\mathrm{k} 1 \mathrm{CA}+\mathrm{k} 2
$$

$\mathrm{k} 1, \mathrm{k} 2$ - calculated coefficients.

So, for spirographic indicators, there are generally accepted formulas for calculating the average population values of indicators depending on the age and height of a person. But this scoring method does not take into account the different significance of the indicators for the BA score. It is natural to consider its correlation coefficient $(\mathrm{R})$ with age as a measure of the indicator's significance. Therefore, the further development of the method is to take these correlation coefficients into account when calculating BA. Then the formulas for calculating the aging rate and BA will be as follows:

$$
\mathrm{AR}=\Sigma(\mathrm{R}(\mathrm{i}) \times \mathrm{I}(\mathrm{i}) / \mathrm{Im}(\mathrm{i})) / \Sigma \mathrm{R}(\mathrm{i}) ; \mathrm{BA}=\mathrm{CA} \times \mathrm{AR}
$$

Here is an example of calculating the BA of a man aged 60 years, whose systolic blood pressure (SBP) is $160 \mathrm{~mm} \mathrm{Hg}$, and the erythrocyte sedimentation rate (ESR) is $12 \mathrm{~mm} / \mathrm{h}$. First, you need to calculate the population mean SBP and ESR for a 60-year-old man using the regression formulas obtained in advance. Let SBP for 60 years be $150 \mathrm{~mm} \mathrm{Hg}$ and ESR $10 \mathrm{~mm} / \mathrm{h}$. It is also predetermined that the correlation coefficients with age for SBP and ESR are 0.3 and 0.4 , respectively. Then, AR $=(0.3 \times 160 / 150+$ $0.4 \times 12 / 10) /(0.3+0.4)=1.14$, and $\mathrm{BA}=60 \times 1.14=68$ years.

The aim of the study was to compare the accuracy of the BA estimate by the multiple regression method and the alternative method.

\section{Materials and methods}

To compare different methods for calculating BA, data from a survey of 110 healthy people aged 50-80 years were used. The following indicators were determined:

SBP - systolic blood pressure, $\mathrm{mm} \mathrm{Hg}$;

DBP - diastolic blood pressure, $\mathrm{mm} \mathrm{Hg}$;

$\mathrm{PBP}=\mathrm{SBP}-\mathrm{DBP}, \mathrm{mm} \mathrm{Hg} ;$

ESR - erythrocyte sedimentation rate, $\mathrm{mm} / \mathrm{hour}$;

$\mathrm{AI}$ - atherogenic index;

$\mathrm{Cr}$ - creatinine, $\mu \mathrm{mol} / \mathrm{l}$

PI - prothrombin index, \%. 
The normal distribution of indicators was checked. It turned out that all indicators, except for ESR, have a normal distribution. After taking the ESR logarithm, its distribution became normal.

Statistical processing of the obtained data was carried out with the help of Excel 2007 and Statistica 7 programs. Standard statistical procedures, including variation and regression analyses, were used.

\section{Results and discussion}

Based on the data from a survey of healthy people, a multiple regression equation was calculated that connects the values of the indicators and the age of people $(R=0.64 ; \mathrm{p}<0.0001)$ :

$$
\text { BA=0,051 PBP+5,98 LOG10(ESR)+0,099 Cr+0,66 AI-0,28 PTI }
$$

The ratio between CB and BA calculated by formula (4) is shown in Fig. 1. The standard error of the BA-CA differences was 4.6 years. This is good model accuracy. This is a typical error in estimating BA using a multiple regression equation, which is a mathematical artifact [4].

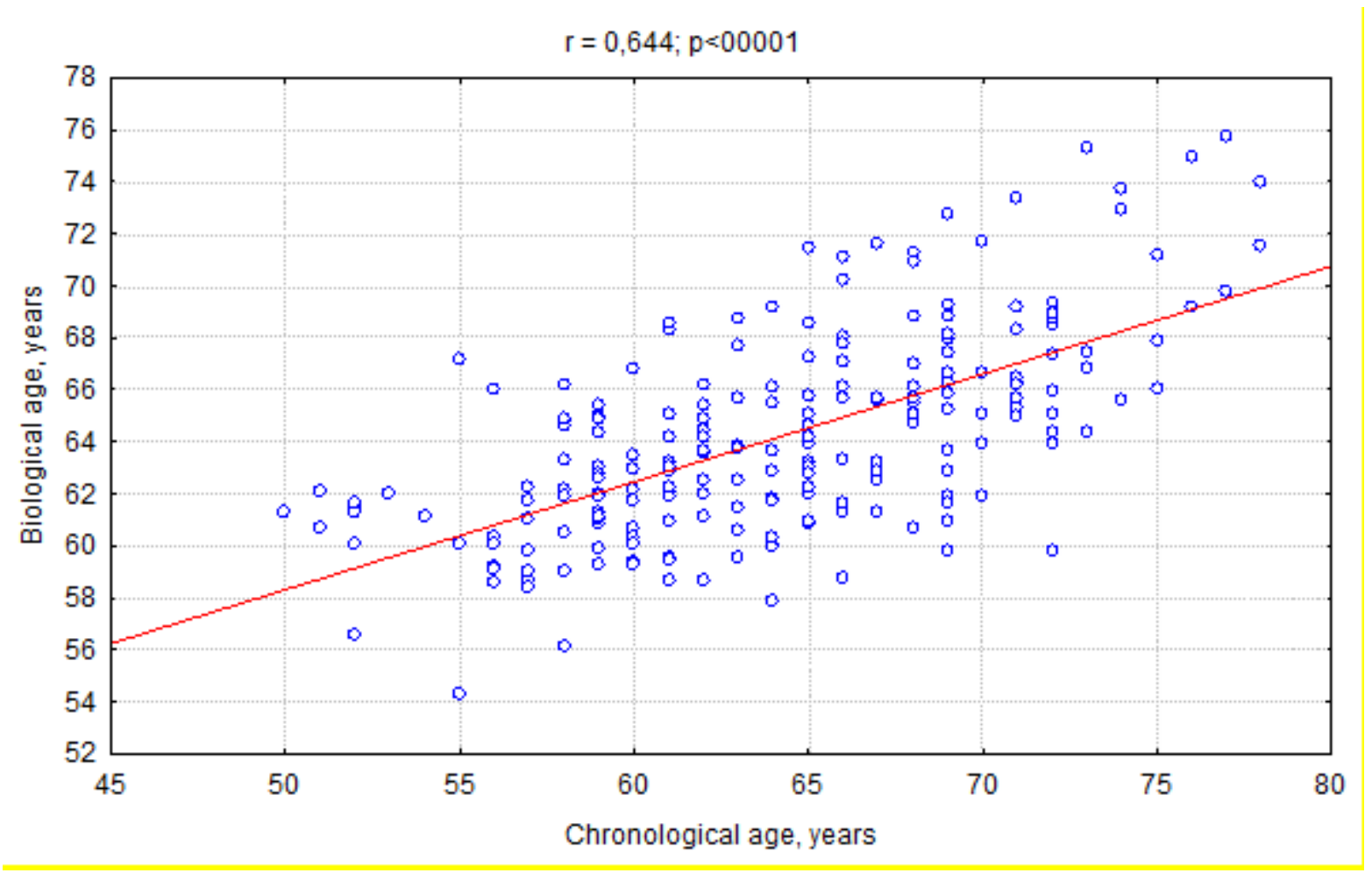

Figure 1. Correlation between biological age and chronological age (regression method).

The dependence of the absolute error in determining the age on the value of the chronological age is shown in Fig. 2. For people under 65, the estimated age is greater than that observed, and for people over 65, it is less. 


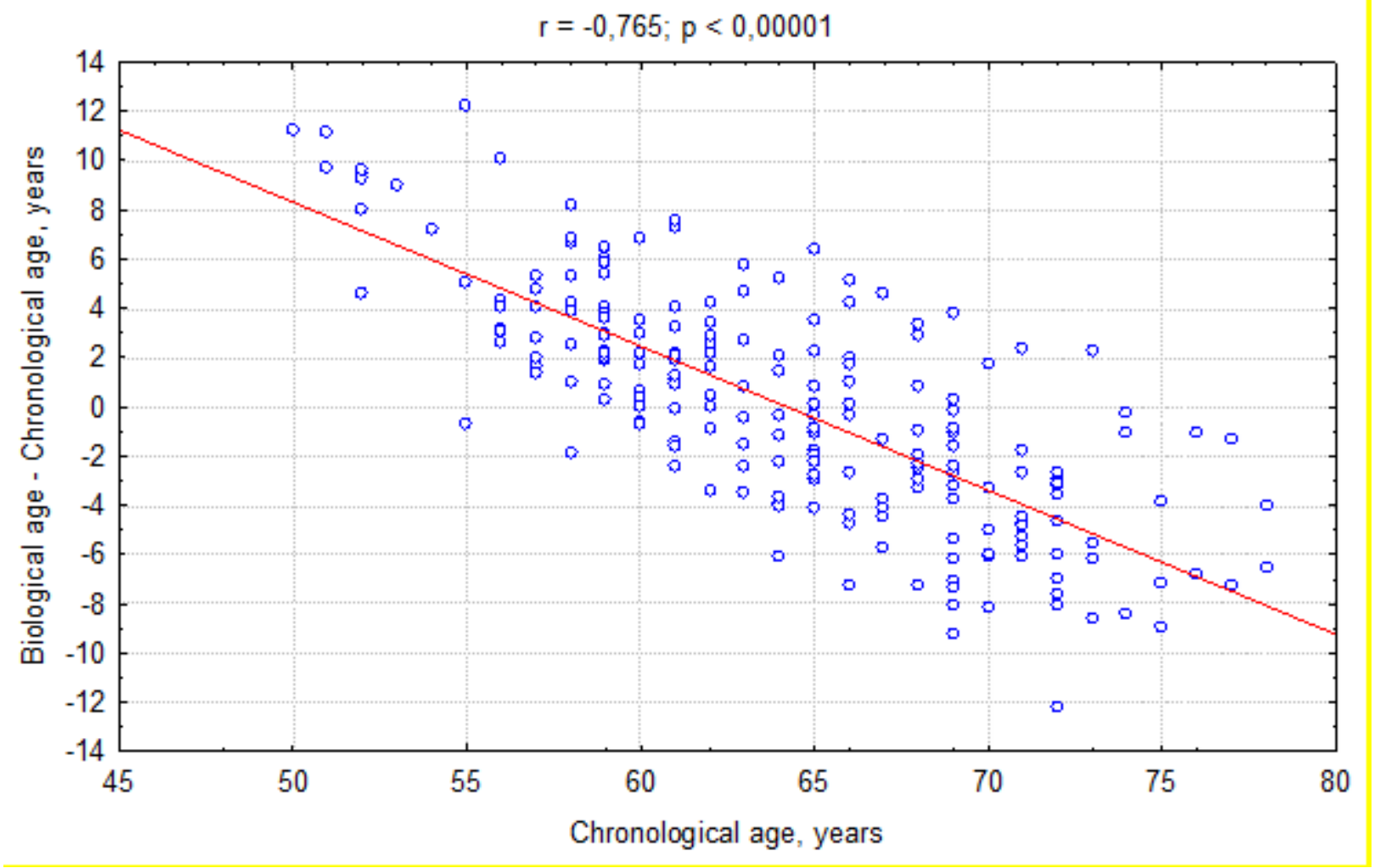

Figure 2. Correlation between chronological age and differences among biological and chronological age (regression method).

An alternative method described above is suggested to fix this error. To implement this method, the coefficients of linear regression equations were previously calculated, linking the values of each BM of aging with the age of the examined people (Tab.). These equations allow calculating the average values of indicators (Im) for each age, in order to then compare them with the values of indicators for a particular person.

Table

Values of coefficients of equations of linear regression of indicators with age

\begin{tabular}{|l|c|c|}
\hline \multicolumn{1}{|c|}{ Biological marker of aging } & k1 & k2 \\
\hline SBP-DBP, $\mathrm{mm} \mathrm{Hg}$ & 0.68 & 19.9 \\
\hline LOG10(ESR), mm/h & 0.02 & 0.43 \\
\hline Atherogenic index & 0.06 & 0.013 \\
\hline Creatinine, mkmol/l & 0.71 & 16.48 \\
\hline PI, \% & -0.558 & 125 \\
\hline
\end{tabular}

The group-average values of indicators for each person were calculated based on the data given in the table. Then the BA of each person was calculated using the formula (1). The standard error of the BA-CA differences was 6.3 years in this case. The use of the improved formula (3) made it possible to reduce the error to 5.5 years. This is slightly more than the multiple regression calculation gave.

However, as follows from Fig. 3-4, the multiple correlation value is higher $(0.758 ; \mathrm{p}<00001)$ and the BA calculation error does not depend on CB. 


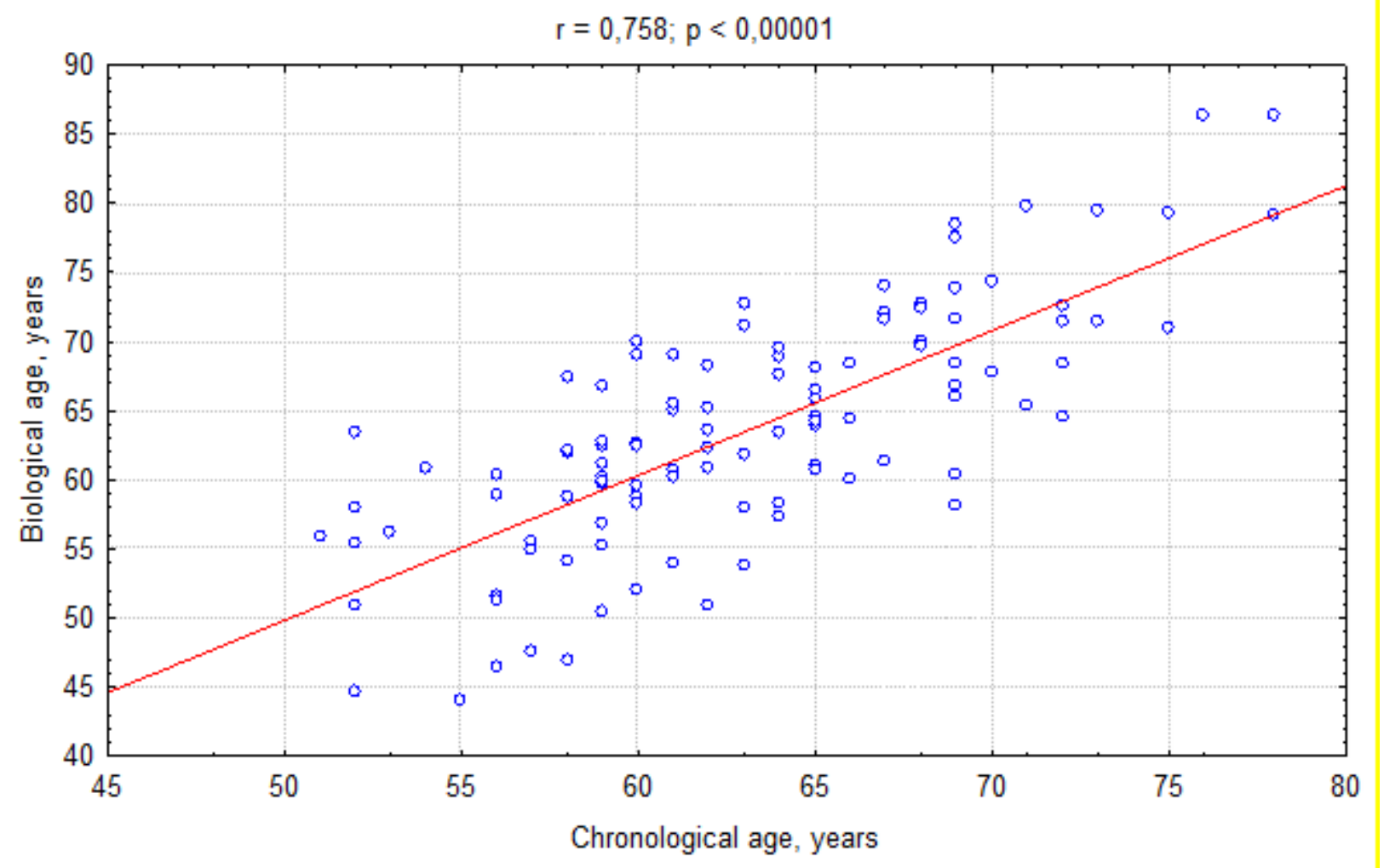

Figure 3. Correlation between biological age and chronological age (alternative method).

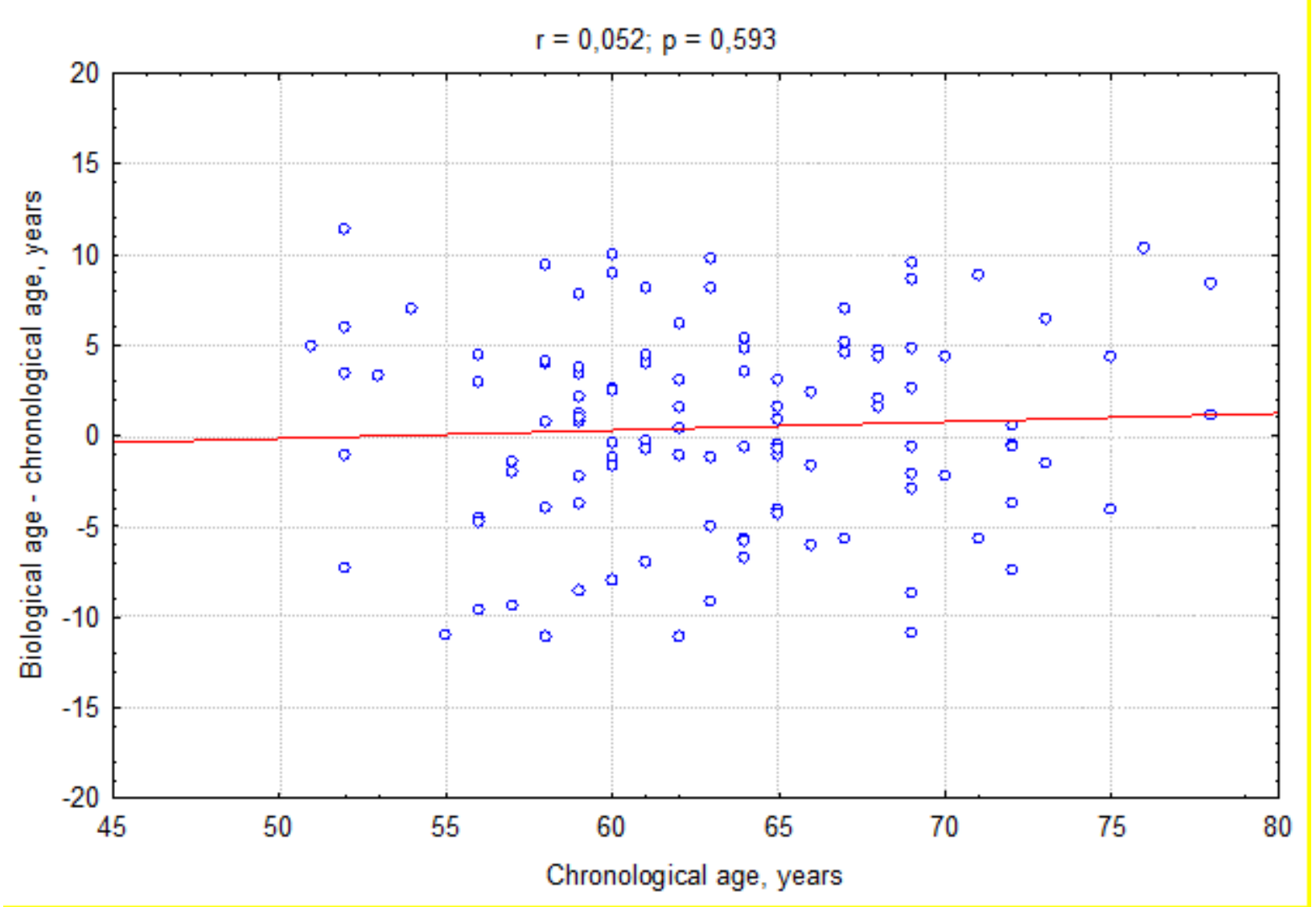

Figure 4. Correlation between chronological age and differences among biological and chronological age (alternative method). 
BA calculation was performed using an alternative method based on incomplete initial data - PI was not used. The standard error of the BA-CA differences was larger and amounted to 7.5 years in this case. Thus, our proposed alternative mathematical method for calculating BA has a sufficiently high accuracy and is devoid of the drawbacks of the method based on the multiple regression equation.

\section{Information about Author:}

Anatoly V. Pisaruk - DSc (Medicine), Head of the Laboratory for Mathematical Modeling of Aging Processes; https://orcid.org/0000-0001-5522-0172 


\section{References}

1. Anstey, K.J.; Lord, S.R.; Smith, G.A. Measuring human functional age: a review of empirical findings. Exp Aging Res 1996, 22, pp 245-266. doi: 10.1080/03610739608254010

2. Aykroyd, R.G.; Lucy, D.; Pollard, A.M.; Solheim, T. Technical note: regression analysis in adult age estimation. Am J Phys Antropol 1997, 104, pp 259-265. doi: 10.1002/(SICI)10968644(199710)104:2<259::AID-AJPA11>3.0.CO;2-Z

3. Singha, B.; Krishana, K.; Kaura, K.; Kanchanb, T. Stature estimation from different combinations of foot measurements using linear and multiple regression analysis in a North Indian male population. J Forensic Leg Med 2019, 62, pp 25-33. doi: 10.1016/j.jflm.2018.12.007

4. Krut'ko, V.N.; Smirnova, T.M.; Dontsov, V.I.; Borisov, S.E. Diagnosing aging: I. Problem of reliability of linear regression models of biological age. Human Physiol 2001, 27, pp 725-731.

5. Krøll1, J.; Saxtrup, O. On the use of regression analysis for the estimation of human biological age. Biogerontology 2000, 1, pp 363-368.

6. Kumari, K.; Yadav, S. Linear regression analysis study - Journal of the practice of Cardiovascular. Sciences 2018, 4, pp 33-36. doi: 10.4103/jpcs.jpcs_8_18

7. Reshetyuk, A.L. Determination of functional age and rates of human aging (in Ukrainian). Ed. Reshetyuk, A.L.; Polyakov, O.A; Korobeinikov. Methodical recommendations Ministry of Health of Ukraine: Kyiv, 1996, p.8 